\title{
Transparent conducting film: Effect of vacuum filtration of carbon nanotube suspended in oleum
}

\author{
TSUYOSHI SAOTOME*, HANSANG KIM, ZHE WANG, DAVID LASHMORE ${ }^{\dagger}$ and \\ H THOMAS HAHN ${ }^{\dagger \dagger}$ \\ UCLA Mechanical and Aerospace Engineering Department, Multifunctional Composites Laboratory, \\ Los Angeles CA 90095, USA \\ ${ }^{\dagger}$ Nanocomp Technologies, Inc., 162 Pembroke Road, Concord, NH 03301 \\ ${ }^{\dagger \dagger}$ California Nano Systems Institute, 570 Westwood Plaza, Building 114, Los Angeles CA 90095, USA
}

MS received 25 July 2009

\begin{abstract}
Vacuum filtration process to fabricate a transparent conducting carbon nanotube (CNT) film is reported. A CNT mat, which is a fibrous sheet of long multi-walled carbon nanotubes (MWNT), was prepared and dispersed in oleum by solution-sonication. The suspension was then vacuum filtered to obtain a thin MWNT layer with improved dispersion. Sheet resistance of the obtained MWNT layer was increased despite the improved dispersion. SEM micrographs and energy dispersive spectroscopy results indicated that the increase of the sheet resistance could be attributed to degradation and oxidation of the MWNT bundles. Though the chemical approach in this study did not improve the electrical property of the CNT mat, a mechanical approach proposed in our recent work was deemed suitable to enhance optical and electrical properties of the CNT mat.
\end{abstract}

Keywords. Transparent conducting film; carbon nanotube; oleum.

\section{Introduction}

Transparent conducting (TC) films made of carbon nanotubes (CNT) (Iijima 1991) have been widely studied for computer displays, touch panels, and solar cells applications (Sreekumar et al 2003; Hu et al 2004; Wu et al 2004; Zhang et al 2005; Zhou et al 2006). As compared to conventional indium tin oxide (ITO), there are a few advantages of using CNT for TC films including: (i) The entangled CNT networks offer higher structural flexibility and robustness under a large deformation than fragile ITO (Zhang et al 2005). (ii) CNT is currently expensive to synthesize, though high-purity CNTs are expected to be mass-produced in the near future (Hata et al 2004). Considering the limited availability of indium to fabricate ITO, CNT should be a much more convenient material to obtain. (iii) Also, ITO, as compared to CNT, requires costly equipment for its fabrication.

There are two approaches to improve the electrical conductivity of CNT TC film while maintaining its visible light transmittance: (i) Improve the CNT dispersion, or make the CNT bundle size smaller so that each CNT can contribute more to transport electrons (Zhou et al 2006). (ii) Use longer CNT to reduce the number of

\footnotetext{
*Author for correspondence (saotome@naa.att.ne.jp)
}

contact points which otherwise increase the resistance (Hu et al 2004; Zhang et al 2005).

In this study, a CNT mat, which is a fibrous sheet of relatively long multi-walled carbon nanotubes (MWNT) (maximum length is $1 \mathrm{~mm}$ ), was prepared. Due to the length of the MWNT, the CNT mat already had relatively low sheet resistance $(302 \Omega / \square$ when visible light transmittance at $550 \mathrm{~nm}$ is $\mathbf{5 6 . 5 \%}$ (Saotome et al 2011). The purpose of this paper is to investigate if the electrical property of the CNT mat can be further enhanced by improving the MWNT dispersion through a vacuumfiltration process.

\section{Experimental}

\subsection{Sample preparation}

A CNT mat with long multi-walled carbon nanotubes (MWNT) network was prepared by gas phase pyrolysis deposition on a moving belt by Nanocomp Technologies, Inc. (Saotome et al 2011). The CNT mat was dispersed in oleum (Sigma Aldrich, 323551: sulfuric acid with 20\% sulfur trioxide) using sonication in an ultrasonic bath (Bransonic, ultrasonicator 1510R-DTH: $70 \mathrm{~W}$ output) under $60^{\circ} \mathrm{C}$ for $15 \mathrm{~min} ., 1 \mathrm{~h}$, and $4 \mathrm{~h}$. Oleum was chosen as a solvent because it is a strong electrophile and should charge MWNT positively to form repulsive forces among 

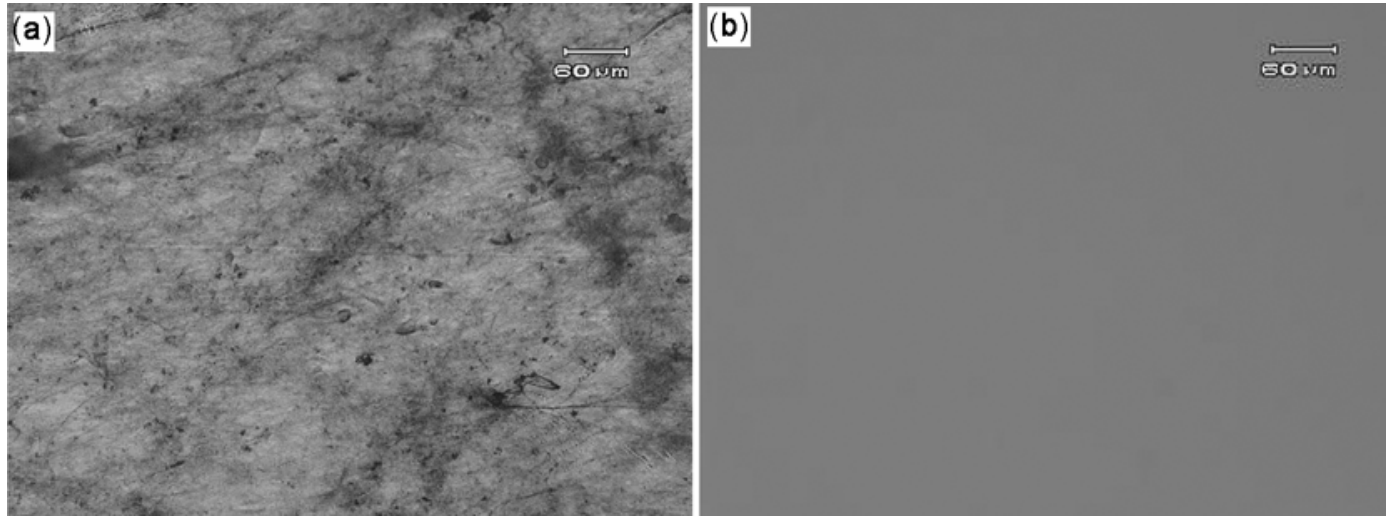

Figure 1. As-received CNT mat (a) showing good dispersion after solution-sonicated in oleum for an hour $(\mathbf{b})$.

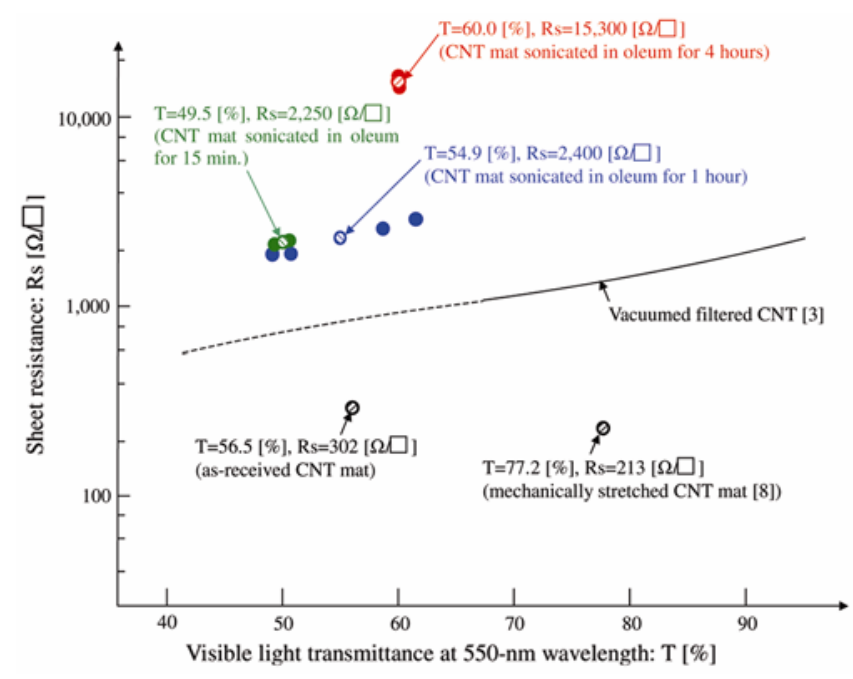

Figure 2. Sheet resistance and visible light transmittance at $550 \mathrm{~nm}$ wavelength of vacuum-filtered MWNT layer (solutionsonicated in oleum for $15 \mathrm{~min}, 1 \mathrm{~h}$ and $4 \mathrm{~h}$ ). Data for as-received CNT mat, mechanically stretched CNT mat (Saotome et al 2011) and vacuum-filtered CNT (a black curve) (Hu et al 2004) are also shown as a reference.

each MWNT (Sreekumar et al 2003). This repulsive interaction was predicted to improve MWNT dispersion (Sreekumar et al 2003). The CNT mat was dispersed in oleum by solution-sonication, and then the solution was vacuum-filtered to obtain a thin MWNT layer. MWNT layer with long fibers and improved dispersion was expected to offer enhanced conductivity. It was confirmed that our CNT mat exhibited good dispersion after solution-sonicated in oleum for $1 \mathrm{~h}$, figure 1 . We also performed a solution-sonication of the CNT mat in deionized water (DI water) with $1 \mathrm{wt} \%$ sodium dodecyl sulfate (SDS) and dimethylformamide (DMF) under the same sonication power $(70 \mathrm{~W})$, temperature $\left(60^{\circ} \mathrm{C}\right)$, and time $(1 \mathrm{~h})$. Though both solutions are widely used to obtain good CNT dispersion, our CNT mat did not dis- perse at all in both solutions, but formed huge agglomerations which could be observed by visual inspection. This difficulty in dissolution is due to the long MWNT fibres of the CNT mat. After the solution-sonication, $10 \mathrm{ml}$ of oleum with MWNT suspension was carefully poured into $150 \mathrm{ml}$ of DI water for dilution. This process was required because filtered MWNT layer had to be rinsed with DI water to wash out sulfuric acid. When MWNT suspended in oleum was deposited on a filter without dilution, the remaining oleum in the MWNT layer reacted with DI water to form sulfuric acid and damaged the MWNT layer during the rinsing process. The diluted oleum with MWNT suspension was vacuum-filtered onto poly(vinylidene fluoride) (PVDF) filter (Millipore, IPVH00010: $450 \mathrm{~nm}$ pore size). Before the suspension was completely filtered, DI water was gently added to rinse out the remaining sulfuric acid until the $\mathrm{pH}$ level of the solution reached 7 (neutralized). PVDF filter was used because it was much easier to take out the deposited MWNT layer, after it was dried, than taking out the MWNT layer deposited on an alumina filter (Whatman, Anodisc 47, $20 \mathrm{~nm}$ pore size). Poly tetrafluoroethylene (PTFE) with a surface energy of $23.9 \mathrm{dyne} / \mathrm{cm}$ should have offered even less MWNT adhesion than PVDF filter which had a surface energy of 33.2 dyne/cm (Brandrup et al 1999). But, since it is difficult to wet PTFE with methanol, which easily wets PVDF, we chose PVDF for the vacuum filtration process. After completing the vacuum filtration, dried MWNT layer was transplanted to a polyethylene terephthalate (PET) film with an adhesive on a backside (Teraoka Seisakusyo Co Ltd) to measure the visible light transmittance.

\subsection{Sample testing}

Visible light transmittance and sheet resistance of the vacuum-filtered MWNT layer were measured. Visible light transmittance of the MWNT layer transplanted to 

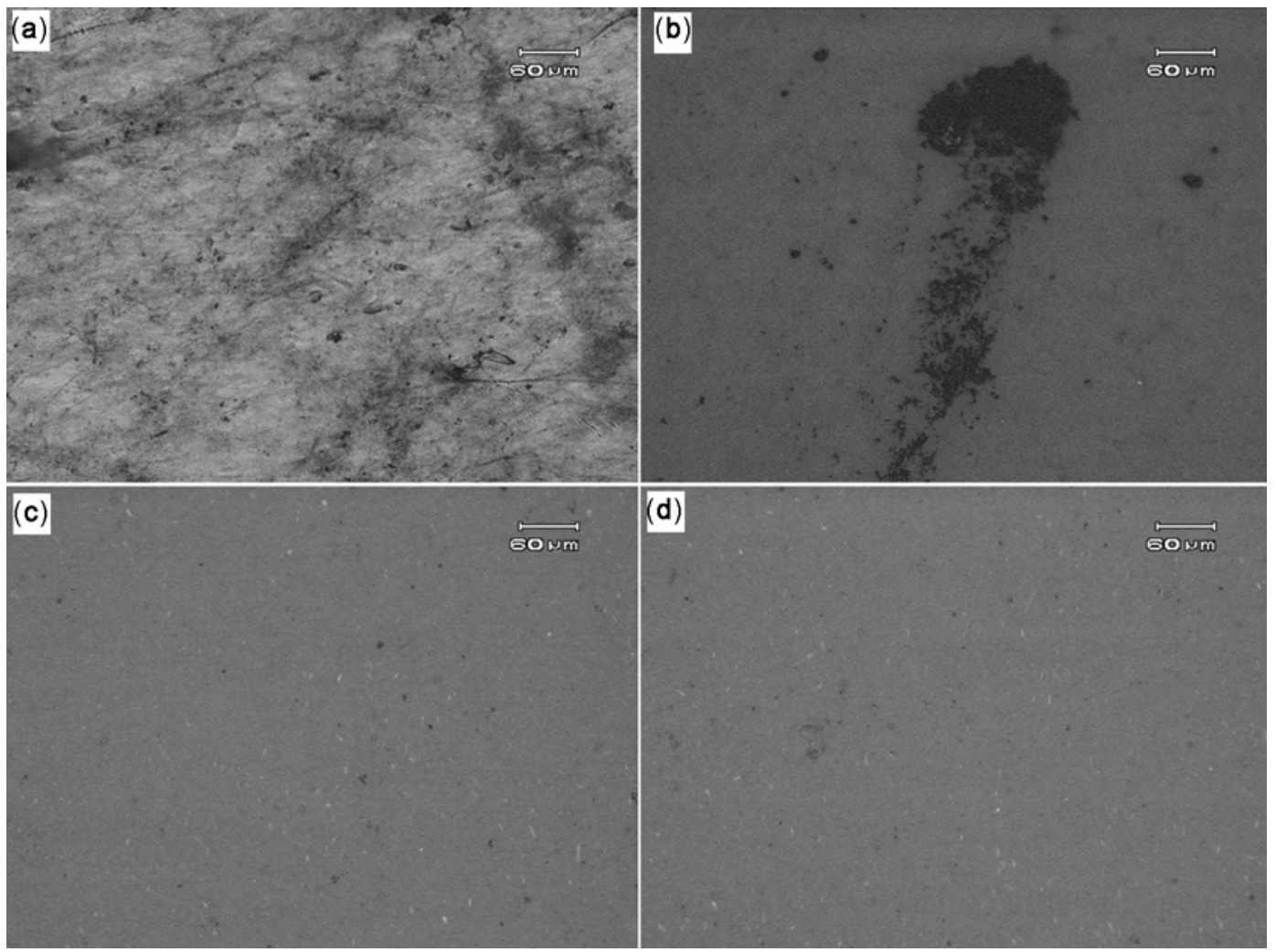

Figure 3. Optical micrographs of (a) as-received CNT mat, and vacuum-filtered MWNT layer solutionsonicated in oleum for (b) $15 \mathrm{~min}$, (c) $1 \mathrm{~h}$ and (d) $4 \mathrm{~h}$. Large agglomerations are observed after $15 \mathrm{~min}$. sonication, whereas much smaller agglomerations are observed after $1 \mathrm{~h}$ or longer sonication.

the aforementioned PET film was measured at $550 \mathrm{~nm}$ wavelength by ultraviolet-visible spectroscopy (UV-Vis). A pristine PET film was used as the background. 550-nm wavelength was chosen for a clear comparison with our previous work (Saotome et al 2011). The PVDF with MWNT layer was cut in a rectangular shape and sheet resistance was measured in the manner that we previously performed (Saotome et al 2011).

\section{Results and discussion}

Figure 2 shows sheet resistance and visible light transmittance at $550 \mathrm{~nm}$ wavelength of vacuum-filtered MWNT layers. Each MWNT went through the solution-sonication in oleum for $15 \mathrm{~min}, 1 \mathrm{~h}$ and $4 \mathrm{~h}$ respectively. Data for as-received CNT mat, mechanically stretched CNT mat (Saotome et al 2011), and vacuum-filtered CNT by black curve (Hu et al 2004) are also shown for comparison. Our vacuum-filtered samples show increased sheet resistance as compared to the as-received CNT mat regardless of the sonication time.

Optical micrographs of as-received CNT mat and vacuum-filtered MWNT layers are shown in figure 3. Large agglomerations are observed after 15 min sonication, whereas much smaller agglomerations could be observed after $1 \mathrm{~h}$ sonication. When agglomerations are formed, they do not contribute significantly to carry electrons and thus decrease the electrical conductivity (Zhou et al 2006). The big agglomerations should be the main reason for the increased sheet resistance after $15 \mathrm{~min}$ sonication.

However, sheet resistance of the vacuum-filtered MWNT with $1 \mathrm{~h}$ and $4 \mathrm{~h}$ sonication was also higher compared to the as-received CNT mat, although the MWNT layers showed much more uniform dispersion than asreceived CNT mat (figure 3). There appear to be three reasons to explain the phenomenon: (i) formation of sheet-like, carbonaceous segments; (ii) oxidation of the MWNT and the carbonaceous segments; and (iii) shortening of the MWNT.

Figure 4 shows scanning electron microscopy (SEM) images of as-received CNT mat and the MWNT layers after the solution-sonication. Sheet-like structures are observed in both $1 \mathrm{~h}$ and $4 \mathrm{~h}$ sonication cases and the structures grow larger as sonication proceeds. Figure 4(b) clearly shows that MWNT's entangled network is covered by the sheet-like structures. In figure 4(c), most of the web-like MWNT network is replaced by the sheetlike structures. Iijima et al (1991) observed similar structures when single-walled carbon nanotubes (SWNT) were solution-sonicated in a mixture of sulfuric and nitric acid (3:1) (Zhang et al 2000). They claimed that the sheet-like 

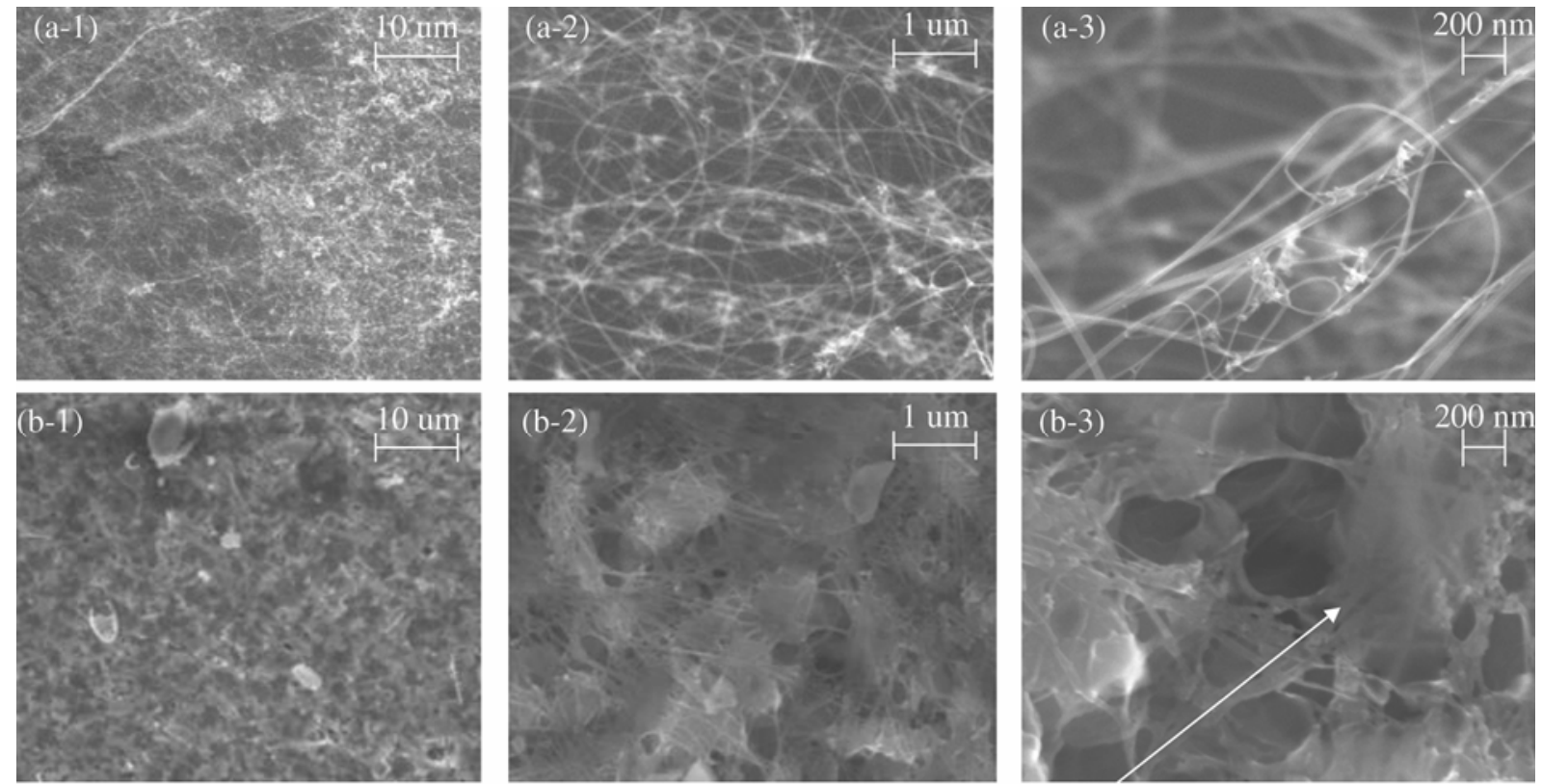

MWNT network covered by a sheet-like structure.
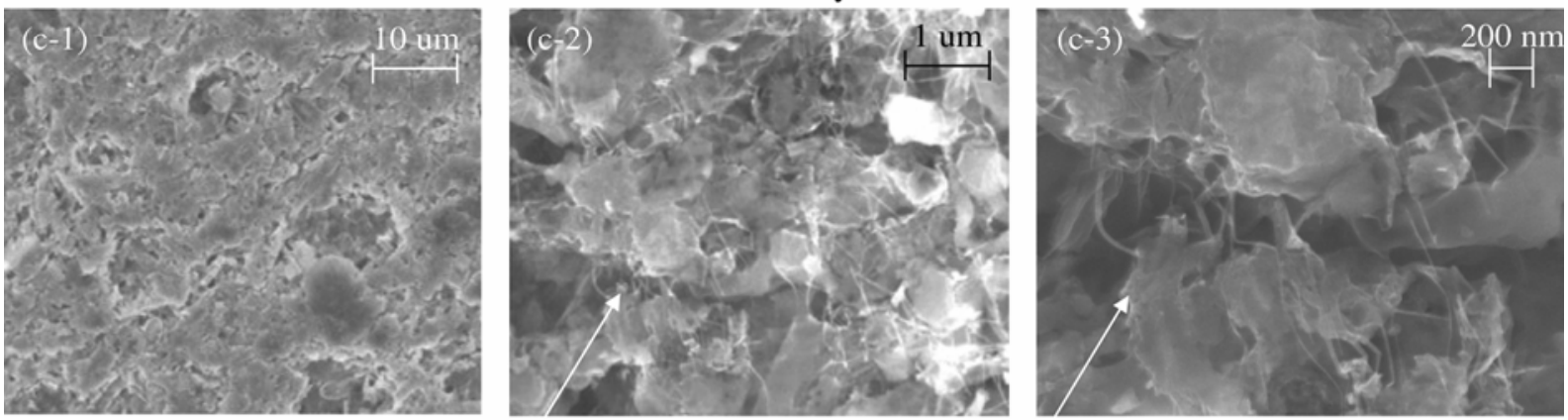

Most of the web-like MWNT network was replaced by the sheet-like structures

Figure 4. Scanning electron microscopy (SEM) images of: (a) as-received CNT mat, (b) vacuum-filtered MWNT layer with 1-hour sonication in oleum, and (c) vacuum-filtered MWNT layer with $4 \mathrm{~h}$ sonication in oleum. Sheet-like structures are observed after $1 \mathrm{~h}$ sonication and they grow larger as sonication proceeds.

structures were carbonaceous segments generated from the SWNT synthesis process as contaminants and part of SWNT fibres decomposed during the solution-sonication in the acid, combined to form large chunks (Zhang et al 2000). Haddon et al also reported that SWNT solutionsonicated in nitric acid decomposed to form a lot of carbonaceous segments ( $\mathrm{Hu}$ et al 2003). From the close resemblance of the morphologies, we believe that the MWNT network in our sample went through the same degradation process to form the carbonaceous segments and resulted in the increased sheet resistance.

Atomic compositions of the as-received CNT mat and oleum-processed MWNT layer were analyzed by energy dispersive spectroscopy (EDS) (figure 5). Each sample was exposed to $5 \mathrm{keV}$ electron beam for the analysis. The results show that as-received CNT mat consisting primarily of carbon structures acquire more oxygen atoms as sonication in oleum is prolonged: the initial oxygen content of $18.9 \%$ increases to $39.6 \%$ after $1 \mathrm{~h}$ sonication and then further to $68.5 \%$ after $4 \mathrm{~h}$ sonication. It was reported that when SWNT was mixed with oleum at $60^{\circ} \mathrm{C}$ and stirred for a certain amount of time, oxidized functional groups such as $\mathrm{C}-\mathrm{OH}$ and $\mathrm{COOH}$ were formed (Sreekumar et al 2003). Formation of these functional groups converts $\pi$-conjugated and thus conductive carbon structures to non-conductive, $s p^{3}$-hybridized carbon structures, and reduces the electrical conductivity. Fogden et al (2008) suggested that CNT acid purification process sometimes caused the carbon contaminants such as amorphous carbons to be oxidized, instead of being burned and eliminated (Fogden et al 2008). The large content of oxygen we observed suggests that the sheet-like structure can be 'oxidized carbonaceous segments'.

It is also known that sonication of CNT in a strong acid such as sulfuric and nitric acid mixture can cut CNT (Liu et al 1998; Zhang et al 2000) and decrease the electrical conductivity. Though we could not directly observe any MWNTs shortened through the sonication process, it is 

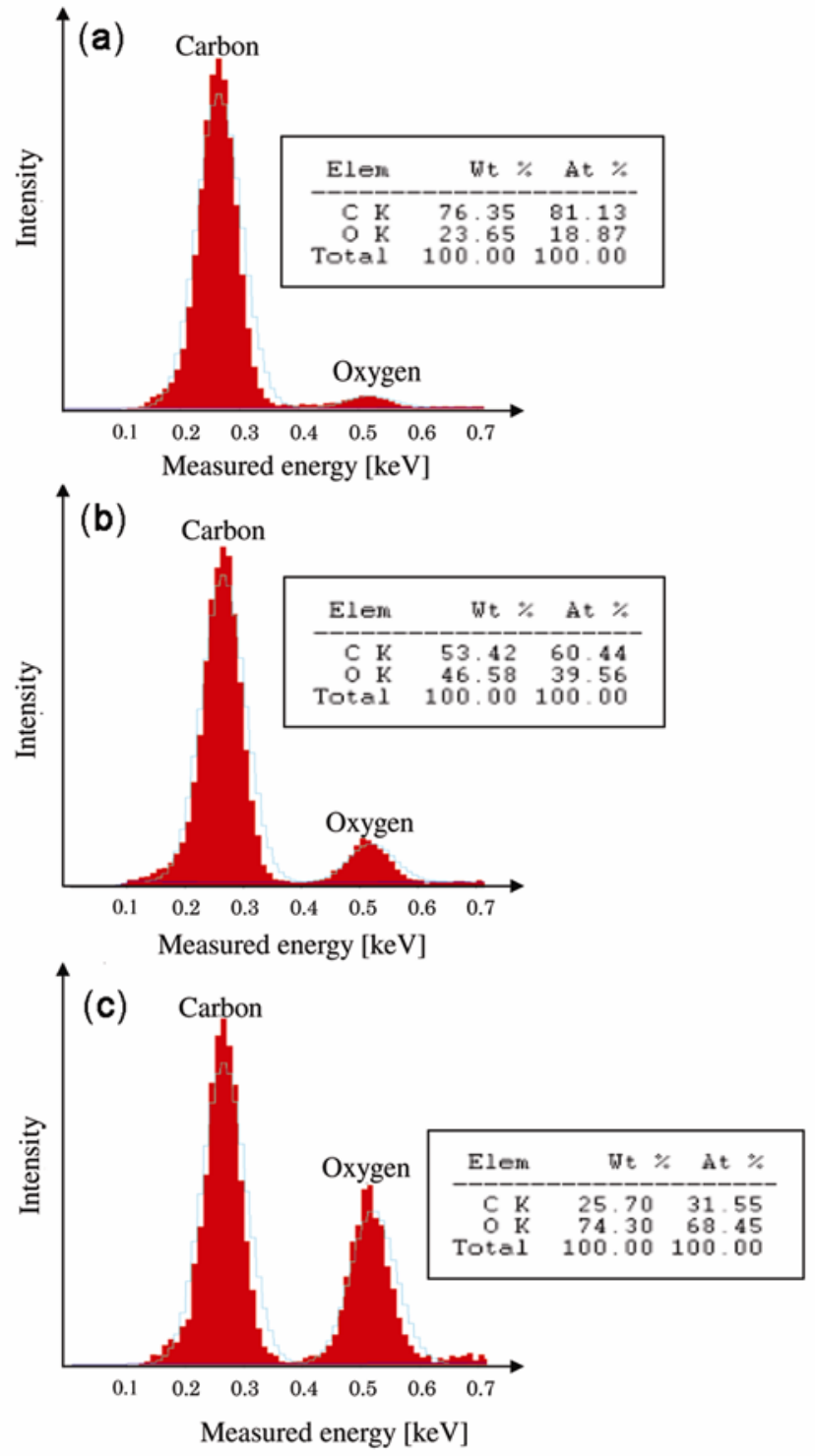

Figure 5. Atomic compositions of (a) as-received CNT mat and MWNT layer sonicated in oleum for (b) $1 \mathrm{~h}$ and (c) $4 \mathrm{~h}$. Energy dispersive spectroscopy (EDS) was used for the analysis. Each sample was exposed to $5 \mathrm{keV}$ electron beam.

reasonable to think that some of the network was cut short during the sonication process. To confirm the phenomenon, observation of MWNT by high-resolution transmission electron microscopy (TEM) should be performed in our future work.

In our recent study, we used a mechanical approach to successfully improve the sheet resistance of the CNT mat from $302 \Omega / \square$ (as-received) to $213 \Omega / \square$ and the visible light transmittance at $550 \mathrm{~nm}$ wavelength from $56.5 \%$ (as-received) to $77 \cdot 2 \%$ (Saotome et al 2011). Considering the difficulty to reduce the sheet resistance without degrading the MWNT in the chemical approach reported in this study, we conclude that the mechanical approach is suitable to improve the optical and electrical properties of the CNT mat.

\section{Conclusions}

A CNT mat, which is a fibrous sheet of relatively long MWNT, was dispersed in oleum by solution-sonication, and the MWNT suspension was vacuum-filtered to obtain a thin MWNT layer on a PVDF filter. Three different sonication times, $15 \mathrm{~min}, 1 \mathrm{~h}$ and $4 \mathrm{~h}$, were tried. The sheet resistance of the obtained films increased in all three cases. Increased sheet resistance of the MWNT layer solution-sonicated for $15 \mathrm{~min}$ can be explained by huge agglomerations observed by an optical microscope. After $1 \mathrm{~h}$ and $4 \mathrm{~h}$ sonications, the sheet resistance still increased despite the improved dispersion of the MWNT. There are three reasons to explain the phenomenon: (i) formation of sheet-like, carbonaceous segments; (ii) oxidation of the MWNT and the carbonaceous segments; and (iii) shortening of the MWNT network during the solution-sonication process in oleum.

The purpose of our study was to see if the sheet resistance of our CNT-mat with long MWNT fibres could be reduced when the dispersion was improved. Due to the long fibre length of our CNT mat with tightly entangled CNT network, required solution-sonication time of at least $1 \mathrm{~h}$ caused degradation and oxidation of the MWNT, leading to the increased sheet resistance of the obtained MWNT layer despite the improved dispersion.

A mechanical approach introduced in our recent study (Saotome et al 2011) should be more suitable than the chemical approach in this study to improve optical and electrical properties of the CNT mat.

\section{Acknowledgements}

This work was supported by the Air Force Office of Scientific Research through a MURI grant FA9550-06-10326 to the University of Washington with Dr. B. Les Lee as Program Manager. Appreciation is extended to the NSF Materials Creation Training Program (DGE0654431) for the use of experimental facilities. The authors are grateful to Makoto Sato for providing the PVDF filters with some useful suggestions.

\section{References}

Brandrup J, Immergut E H and Grulke E A 1999 Polymer handbook (Wiley-Interscience Publicaion) 4th edn

Fogden S, Verdejo R, Cottam B and Shaffer M 2008 Chem. Phys. Letts. 460162

Hata K, Futaba D N, Mizuno K, Namai T, Yumura M and Iijima S 2004 Science 3061362

Hu H, Zhao B, Itkis M E and Haddon R C 2003 J. Phys. Chem. B107 13838 
Hu L, Hecht, D S and Gruner G 2004 Nano Letts. 42513 Iijima S 1991 Nature 35456

Liu J, Rinzler A G, Dai H, Hafner J H, Bradley R K, Boul P J, Lu A, Iverson T, Shelimov K, Huffman C B, RodriguezMacias F, Shon Y S, Lee T R, Colbert D T and Smalley R E 1998 Science 2801253

Saotome T, Kim H, Lashmore D and Hahn H T 2011 Bull. Mater. Sci. 34615

Sreekumar T V, Liu T and Kumar S 2003 Chem. Mater. 15175
Wu, Z, Chen Z, Du X, Logan J M, Sippel J, Nikolou M, Kamaras K, Reynolds J R, Tanner D B, Hebard A F and Rinzler A G 2004 Science $\mathbf{3 0 5} 1273$

Zhang Y, Shi Z, Gu Z and Iijima S 2000 Carbon 382055

Zhang M, Fang S, Zakhidov A A, Lee S B, Aliev A E, Williams C D, Atkinson K R and Baughman R H 2005 Science 309 1215

Zhou Y, Hu L and Gruner G 2006 Appl. Phys. Letts. 88 123109 